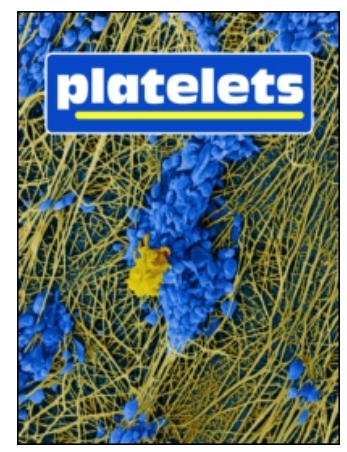

\title{
Platelets
}

\section{Two novel variants of uncertain significance in GP9 associated with Bernard-Soulier syndrome: Are they true mutations?}

P. Boisseau, C. Debord, M. Eveillard, A. Quéméner, M. Sigaud, M. Giraud, P. Talarmain, C. Thomas, G. Landeau, S. Bezieau, B. Pan Petesch, M. C. Béné \& M. Fouassier

To cite this article: P. Boisseau, C. Debord, M. Eveillard, A. Quéméner, M. Sigaud, M. Giraud, P. Talarmain, C. Thomas, G. Landeau, S. Bezieau, B. Pan Petesch, M. C. Béné \& M. Fouassier (2017): Two novel variants of uncertain significance in GP9 associated with Bernard-Soulier syndrome: Are they true mutations?, Platelets, DOI: 10.1080/09537104.2017.1371288

To link to this article: $h$ ttp://dx.doi.org/10.1080/09537104.2017.1371288

Published online: 09 Nov 2017.

Submit your article to this journal ¿

Q View related articles ¿

View Crossmark data $־$ 


\title{
Two novel variants of uncertain significance in GP9 associated with Bernard-Soulier syndrome: Are they true mutations?
}

\author{
P. Boisseau ${ }^{1}$, C. Debord ${ }^{2}$, M. Eveillard ${ }^{2}$, A. Quéméner ${ }^{3}$, M. Sigaud ${ }^{2,4}$, M. Giraud ${ }^{1}$, P. Talarmain ${ }^{1}$, C. Thomas ${ }^{1}$, \\ G. Landeau' ${ }^{1}$, S. Bezieau ${ }^{1}$, B. Pan Petesch ${ }^{5}$, M. C. Béné ${ }^{2}, \&$ M. Fouassier ${ }^{42}$
}

${ }^{7}$ Service de Génétique Médicale, CHU de Nantes, Nantes, France, ${ }^{2}$ Service d'Hématologie Biologique, CHU de Nantes, Nantes, France, ${ }^{3}$ CRCINA, INSERM, CNRS, Université de Nantes, Nantes, France, ${ }^{4}$ Centre Régional de Traitement des Hémophiles, CHU de Nantes, Nantes, France, and ${ }^{5}$ Centre Régional de Traitement des Hémophiles, $\mathrm{CHU}$ de Brest, Brest, France

\section{Abstract}

Bernard-Soulier syndrome (BSS) is an autosomal recessive major thrombocytopathy, the symptoms of which are mainly marked by mucocutaneous bleeding. This rare disease, initially described in the 1970s, is the result of an abnormal formation of the glycoprotein complex lbIX-V (GP Ib-IX-V), a platelet receptor of von Willebrand factor. A large number of mutations, sometimes involving the GP9 gene, have been described as possibly responsible for the disease. We report here the case of a BSS patient who presented with persistent thrombocytopenia $\left(31 \times 10^{9} / \mathrm{L}\right)$ and decreased surface expression of GPIb-IX-V on large platelets with anisocytosis. Thorough molecular analyses disclosed two previously unreported GP9 variants, respectively c.230T>A (p.Leu77GIn) and c.255C >A (p.Asn85Lys). Both are likely to modify the conformation of GP-IX interactions with other glycoproteins of the Ib-IX-V complex and thus proper expression of this complex on the membrane of platelets.
\end{abstract}

\section{Keywords}

Bernard-Soulier syndrome, GP9

\section{History}

Received 24 April 2017

Revised 4 August 2017

Accepted 4 August 2017

Published online 9 November 2017

\section{Case report}

A female patient of Franco-Mauritian origin, born in 1989, was diagnosed with Bernard-Soulier syndrome (BSS) at 3 years of age. At that time, she was scheduled for an adenoidectomy and preoperative assessment disclosed a profound thrombocytopenia consistent with BSS. She remained mildly symptomatic with frequent bruising, rare post-traumatic subcutaneous hematomas of the limbs and occasional gum bleeding. At puberty, she presented with heavy blood loss during menses, which returned to normal abundance after the introduction of oral contraception. Her sole severe hemorrhagic history then was an hemoperitoneum resulting from the rupture of an ovarian cyst in 2015. She became pregnant in 2016 and gave birth to a little boy after receiving preventive platelet transfusions. No hemorrhagic complication occurred during delivery.

The diagnosis of BSS, in thrombocytopenic patients, is based on the examination of platelets' morphology and flow cytometry analysis of membrane glycoproteins. Alterations in platelets' functions can also be investigated in vitro. Ultimately, the study of genes encoding GP Ib-IX-V proteins provides more information on the transmission and mechanistic of the disease [1]. Indeed, in order to improve knowledge about BSS, an International Consortium for the study of BSS has been established, which has recently published a wide spectrum of mutations concerning GPIBA, GPIBB, and GP9. Among these

Color versions of one or more of the figures in the article can be found online at www.tandfonline.com/iplt.

Correspondence: Marc Fouassier, Centre de Traitement de l'Hémophilie - CHU Hôtel-Dieu, 1 place Alexis Ricordeau, Nantes Cedex 144093 ,

France. E-mail: marc.fouassier@chu-nantes.fr mutations, GP9 was found to be involved with $25 \%$ of the reported mutations [2].

In 2017, it was therefore decided to further explore the disease of this patient and also to perform molecular analyses investigating for BSS-related mutations in genes involved in the GP Ib-IX$\mathrm{V}$ complex.

At that time, ongoing thrombocytopenia was confirmed with a platelet count of $31 \mathrm{G} / \mathrm{L}$. Both the mean platelet volume and mean platelet diameter were unassessable because of the presence of platelets' anisocytosis. Indeed, on blood smears, the patient's platelets displayed marked shape and size heterogeneity and giant platelets could be observed. Flow cytometry was used to test quantitatively the surface expression of GPIb-IX-V on platelets using FITC-conjugated anti-CD42b antibody, according to the manufacturer's recommendations (Biocytex, Marseilles, France). This disclosed 1603 sites on the patient's platelets (normal range $38000 \pm 11000$ ). By contrast, CD41 appeared normally expressed on the same platelets at 51706 sites (normal range $51000 \pm 14000$ ) probably because of the marked platelets anisocytosis. Indeed, in 2016, a higher expression of CD41 (84000) was observed in relation to the large size of the platelets.

Platelet agglutination in the presence of ristocetin is normally part of such explorations, showing no agglutination due to the absence of binding between von Willebrand factor and GPIb. Indeed, in 2006, Light Transmission Aggregometry was performed and confirmed the absence of aggregation in the presence of ristocetin only, showing the absence of the Ib-IX complex. Moreover, in 2015, a MAIPA test confirmed the absence of anti-IbIX antibodies, thus excluding that the complex was hidden by anti-platelets antibodies. 

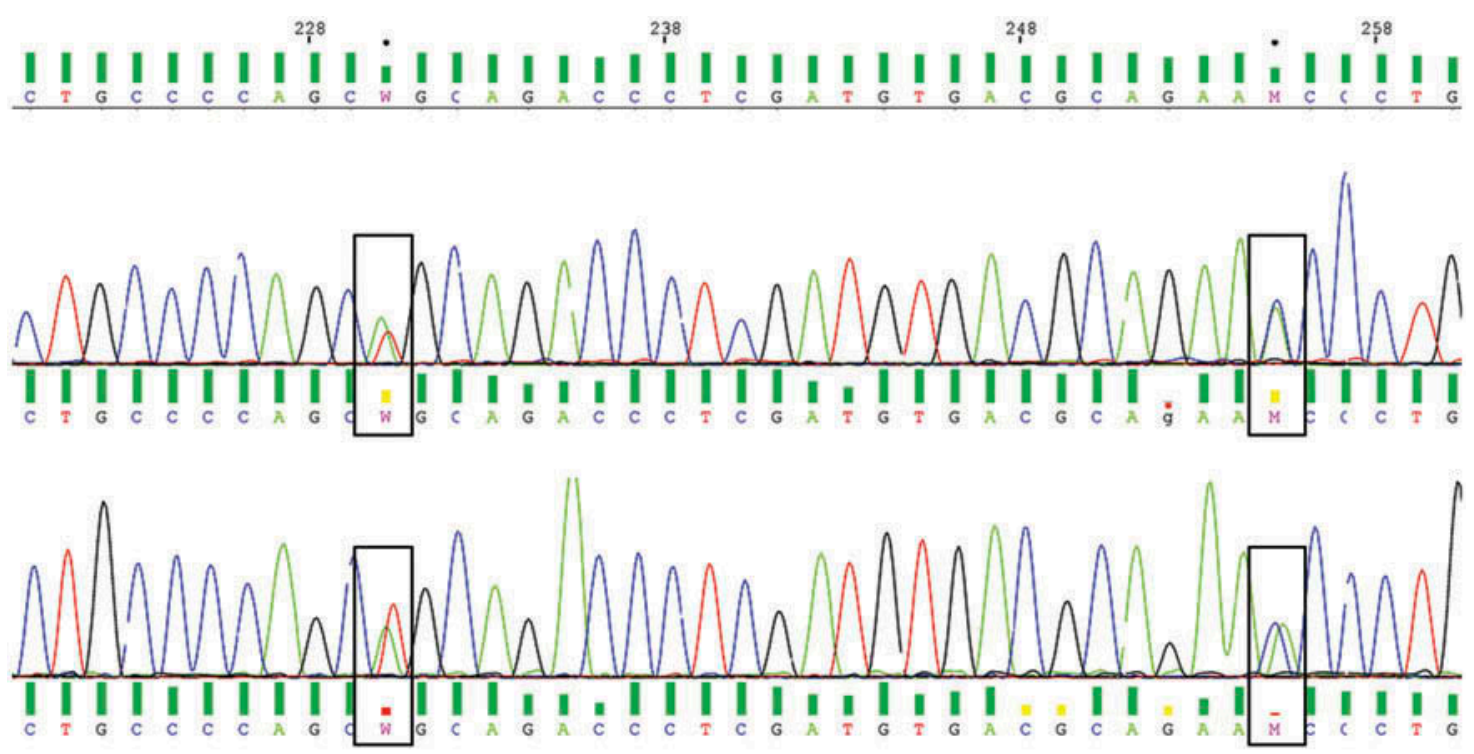

Figure 1. Sanger sequencing electrophoregram showing a composite heterozygous profile for the variants c.230T $>$ A and c.255C $>$ A in GP9 gene.

Molecular genetics analysis of $G P 9, G P 1 B A$, and $G P 1 B B$ by Next Generation Sequencing of the patient's blood DNA disclosed two GP9 previously undescribed variants of uncertain significance, respectively c.230T $>$ A (p.Leu77Gln) and c. $255 \mathrm{C}>\mathrm{A}$ (p.Asn85Lys). The latter were further confirmed by Sanger method (Figure 1). Moreover a bam visualization tool allowed assessing that they were on different alleles.

Finally, pathogenicity was highly suggested by the use of 3 bioinformatics software, respectively Align GVGD (v2007) [3], SIFT (v4.0.3) [4] and MutationTaster (v2013) [5].

\section{Discussion}

The variants identified in this patient involve two amino acids highly conserved in 12 different species, from zebrafish to human [6]. The first, p.Leu77Gln, is located in the LeucineRich Repeat (LRR) domain of the protein. The second, p. Asn85Lys, involves a region at the boundary between the LRR and its C-terminal region. Moreover, each of these variants is likely to induce physicochemical modifications of the protein, when comparing wild type and substituted forms. Leucine is a highly hydrophobic aminoacid, while the substituted glutamine, in p.Leu77Gln, is hydrophilic (Grantham's distance 113 [0-215]). Similarly, the second variant induces a switch from the neutral asparagine to the cationic lysine (Grantham distance 94 [0-215]). Such drastic physicochemical changes would be expected to modify deeply the protein's quaternary structure within the GP Ib-IX-V complex. Moreover, based on the 3D structure of a GPIb $\beta /$ GPIX ectodomain chimera [7], the two residues Leu77 and Asn85 appear completely buried (Figure 2) and therefore certainly contribute to the folding stability of GPIX. Their substitution with hydrophilic or charged amino acids is likely to affect not only the structure of the protein but also interactions with its partners in the formation of the GP Ib-IX-V complex.

The three tools used to test the pathogenicity of the variants predicted a deleterious/disease-causing modification for both variants of uncertain significance. C65 is the maximum level of pathogenicity of Align, a score of 0 with SIFT is also the worse level. For mutation tester, values nearest to 1 for the $\mathrm{p}$ value carry

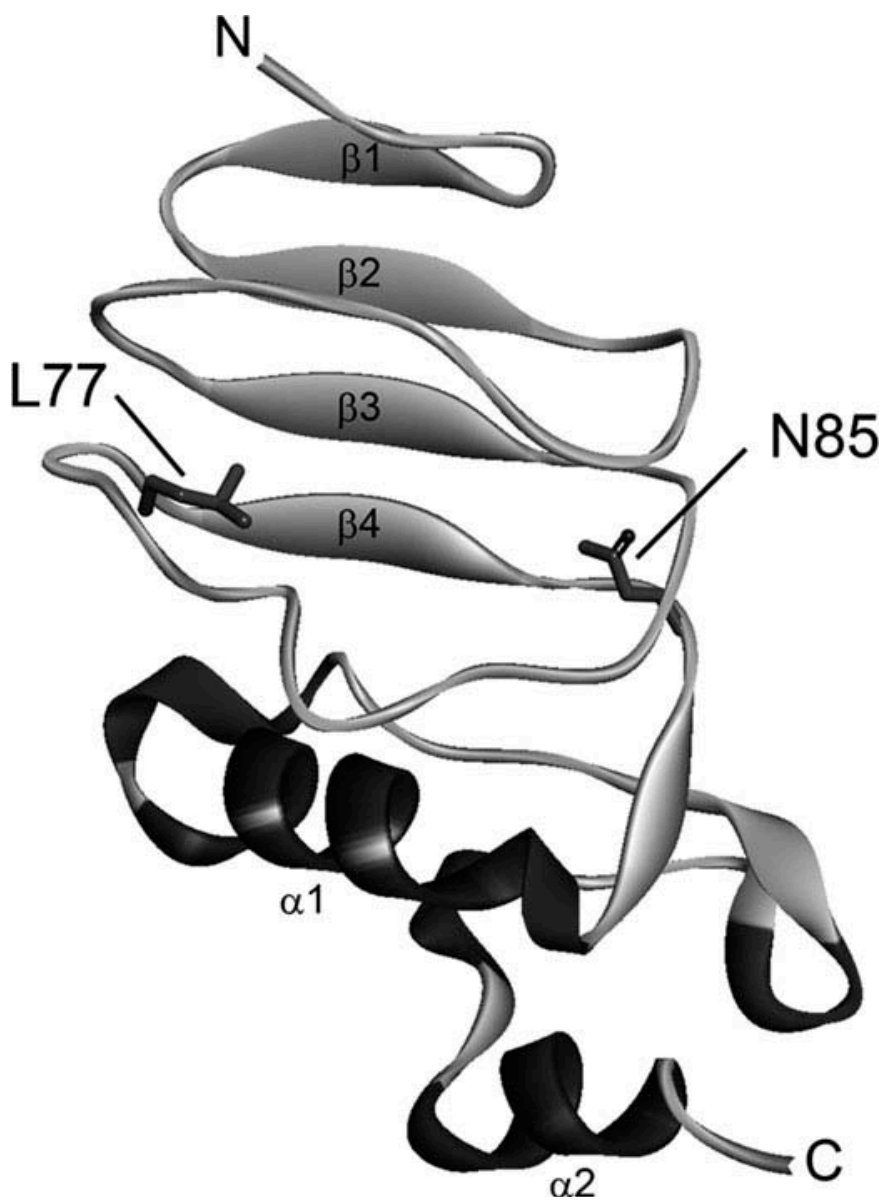

Figure 2. Crystal structure of GPIb?Eabc, a GPIb?/GPIX ectodomain chimera. The structure of a chimera of GPIb? and GPIX ectodomains named GPIb?Eabc (Protein Data Bank code3REZ) in which three non-contiguous sequences that may constitute the putative convex surface of the GPIb? ectodomain are replaced by their GPIX counterparts [4] was used to visualize the position of the conserved residues Leu 77 and Asn 87. Ribbon diagram of the GPIb?Eabc structure (PDB code 3REZ), with Leu residue 77 (L77) and Asn residue 85 (N85) shown as sticks. 
the highest disease-causing probability. These conditions were observed here for both variants with the three tools. All these elements make the deleterious nature of these genetic sequence variants highly probable and mutation seems now to be the most appropriate word to call these variants.

Of interest, interrogation of the panel of the ExAC project, which has tested more than 118500 alleles of healthy volunteers [8] disclosed that these mutations have not been reported previously. This also supports the hypothesis that these sequence variations are likely to have appeared de novo and thus be deleterious.

In summary, analysis of the GP9 gene by molecular studies allowed us to disclose two new variants likely to be responsible for BSS in a mildly symptomatic yet confirmed BSS patient whose disease has been well controlled so far. Even if without direct consequence for the patient's management, these data may help to better understand the structure of the GPIX protein and its link with the GPIb-IX-V complex functions. As reported previously [2], this can involve either the absence of expression of the complex on the platelets' surface, which was confirmed here, or an anomaly in von Willebrand factor binding. Since the first hypothesis seems more likely here, our results highlight the possibility that GPIX's structure is involved in the transport of the complex to the platelets' surface, as do other subunits of the complex, since incomplete polymers or single subunits are degraded and cannot be present isolated on the platelets' surface. Moreover, it has previously reported that mutations in the leucine rich repeat of GP9, which is the case for the two variants disclosed here, are indeed likely to impair correct assembly of the complex [9]. This is consistent with the extremely low level of platelets' surface expression, similar to that reported in the context of a deletion starting at position 86 , also in the vicinity of the leucine-rich repeat of the molecule [9].

\section{Declaration of interest}

The authors report no conflicts of interest.

\section{References}

1. Berndt MC, Andrews RK. Bernard-Soulier syndrome. Haematologica 2011;96:355-359.

2. Savoia A, Kunishima S, De Rocco D, Zieger B, Rand ML, PujolMoix N, Caliskan U, Tokgoz H, Pecci A, Noris P, et al. Spectrum of the mutations in Bernard-Soulier syndrome. Hum Mutat 2014;35:1033-1045.

3. http://www.ngrl.org.uk/Manchester/page/align-gvgd. Last accessed July $21,2017$.

4. http://provean.jcvi.org/about.php. Last accessed july 21, 2017.

5. http://www.mutationtaster.org/. Last accessed july 21, 2017.

6. Aken BL, Achuthan P, Akanni W, Amode MR, Bernsdorff F, Bhai J, Billis K, Carvalho-Silva D, Cummins C, Clapham P, et al. Ensembl. Nucleic Acids Res 2017;4(45):D635-D642.

7. McEwan PA, Yang W, Carr KH, Mo X, Zheng X, Li R, Emsley J. Quaternary organization of GPIb-IX complex and insights into Bernard-Soulier syndrome revealed by the structures of GPIb $\beta$ and a GPIb/GPIX chimera. Blood 2011;118:5292-5301.

8. Lek M, Karczewski KJ, Minikel EV, Samocha KE, Banks E, Fennell T, O'Donnell-Luria AH, Ware JS, Hill AJ, Cummings BB, et al. Analysis of protein-coding genetic variation in 60,706 humans. Nature 2016;536:285-291.

9. Rivera CE, Villagra J, Riordan M, Williams S, Lindstrom KJ, Rick ME. Identification of a new mutation in platelet glycoprotein IX (GPIX) in a patient with Bernard-Soulier syndrome. Br J Haematol 2001;112:105-108. 